\title{
Artesunate inhibits the growth and induces apoptosis of human gastric cancer cells by downregulating COX-2
}

This article was published in the following Dove Press journal:

OncoTargets and Therapy

16 April 2015

Number of times this article has been viewed

\author{
Ping Zhang \\ He-Sheng Luo \\ Ming Li \\ Shi-yun Tan
}

Department of Gastroenterology, Renmin Hospital of Wuhan University, Wuhan, Hubei Province, People's Republic of China
Correspondence: He-Sheng Luo

Department of Gastroenterology, Renmin Hospital of Wuhan University, 238 Jiefang Road, Wuhan, Hubei 430060, People's Republic of China

Tel +86278804 I9II

Fax +86 2788042922

Email luoheshengxhnk@I63.com
Abstract: Artesunate, a derivative of artemisinin isolated from Artemisia annua L., has been traditionally used to treat malaria, and artesunate has demonstrated cytotoxic effects against a variety of cancer cells. However, there is little available information about the antitumor effects of artesunate on human gastric cancer cells. In the present study, we investigated the antitumor effect of artesunate on human gastric cancer cells and whether its antitumor effect is associated with reduction in COX-2 expression. The effects of artesunate on the growth and apoptosis of gastric cancer cells were investigated by 3-(4,5-dimethylthiazol-2-yl)-2,5-diphenyltetrazolium bromide (MTT) assay, flow cytometric analysis of annexin V-fluorescein isothiocyanate/ propidium iodide staining, rhodamine 123 staining, and Western blot analysis. Results indicate that artesunate exhibits antiproliferative effects and apoptosis-inducing activities. Artesunate markedly inhibited gastric cancer cell proliferation in a time- and dose-dependent manner and induced apoptosis in gastric cancer cells a dose-dependent manner, which was associated with a reduction in COX-2 expression. Treatment with the selective COX-2 inhibitor celecoxib, or transient transfection of gastric cancer cells with COX-2 siRNA, also inhibited cell proliferation and induced apoptosis. Furthermore, the treatment with artesunate promoted the expression of proapoptotic factor Bax and suppressed the expression of antiapoptotic factor Bcl-2. In addition, caspase- 3 and caspase- 9 were activated, and artesunate induced loss of mitochondrial membrane potential, suggesting that the apoptosis is mediated by mitochondrial pathways. These results demonstrate that artesunate has an effect on anti-gastric cancer cells. One of the antitumor mechanisms of artesunate may be that its inhibition of COX-2 led to reduced proliferation and induction of apoptosis, connected with mitochondrial dysfunction. Artesunate might be a potential therapeutic agent for gastric cancer.

Keywords: artesunate, gastric cancer cells, COX-2, apoptosis

\section{Introduction}

Gastric cancer is a common malignant tumor in the digestive system, and the morbidity and mortality associated with this disease are ranked second highest of all malignant neoplasms in Eastern Asia, Eastern Europe, and South America. ${ }^{1,2}$ Surgical resection has been regarded as the standard of care for gastric cancer, but the condition in the majority of patients was advanced or metastatic disease at the time of presentation. Thus, chemotherapy plays an integral role in patients with advanced tumors by reducing the mortality of cancer. Anthracyclines, 5-fluorouracil, and cisplatin are chemotherapeutical drugs utilized in gastric cancer treatment. However, the development of drug resistance and severe side effects of standard anticancer drugs limit their therapeutic application. Therefore, the development of more effective and low-toxicity chemotherapeutic agents is an important area of study. 
Cyclooxygenase-2 (COX-2) is constitutively overexpressed in a variety of malignancies, including gastric cancer, breast cancer, bladder cancer, non-small-cell lung cancer, and colorectal cancer, ${ }^{3,4}$ and COX-2 overexpression is associated with carcinogenesis, progression, invasion, metastasis, and a poor prognosis. ${ }^{5-7}$ Therefore, inhibition of COX-2 expression may prevent or reverse gastric carcinogenesis. There is increasing evidence demonstrating that inhibition of expression of COX-2 has antitumor activity against gastrointestinal carcinoma. ${ }^{8}$ However, previous research has revealed that unexpected cardiovascular side effects result when selective COX-2 inhibitors are used in the long term. ${ }^{9}$ Consequently, the development of more effective and low-toxicity selective COX-2 inhibitors is an important area of study.

Artesunate, a derivative of artemisinin isolated from Artemisia annua L., has been approved by the Chinese government for the treatment of malaria, especially against cerebral malaria. Studies demonstrated that it possesses a number of biological activities, including hepatoprotective, antiviral, anti-inflammatory, antioxidative, anti-allergic, antidiabetic, and antibacterial effects. ${ }^{10-15}$ Previous studies have revealed that artesunate could inhibit the proliferation of cells and inhibit angiogenesis in various tumor cell lines in vitro and in vivo, such as breast cancer, lung cancer, colon cancer, pancreatic cancer, and hepatocellular carcinoma. ${ }^{16-18}$ However, there is little available information about the antitumor effects of artesunate on human gastric cancer cells. In the present study, we investigated the antitumor effect of artesunate on human gastric cancer cells and whether its antitumor effect is associated with reduction in COX-2 expression.

\section{Materials and methods Materials}

Gastric cancer cells BGC-823, HGC-27, and MGC-803 were obtained from the Cell Bank of the Shanghai Institute of Biochemistry and Cell Biology, Chinese Academy of Sciences (Shanghai, People's Republic of China). Artesunate was purchased from Guilin South Pharmaceutical Company Limited (purity $>99.0 \%$; Guilin, People's Republic of China). RPMI 1640 medium, fetal bovine serum (FBS), penicillin-streptomycin, pancreatin, glutamine, and a bicinchoninic acid protein assay kit were purchased from Beyotime Institute of Biotechnology (Suzhou, People's Republic of China). An annexin V-fluorescein isothiocyanate (FITC)/ propidium iodide (PI) apoptosis kit was purchased from Hoffmann-La Roche Ltd. (Basel, Switzerland). Rhodamine 123 was purchased from Sigma-Aldrich Co. (St Louis, MO,
USA). A caspase-3 colorimetric assay kit and caspase-9 colorimetric assay kit were obtained from Nanjing Keygen Biotech Company Limited (Nanjing, People's Republic of China). The 2X Taq PCR Master Mix was obtained from Tiangen Biotech Co., Ltd. (Beijing, People's Republic of China). Primers for human COX-2 and $\beta$-actin were designed by Sangon Biotech Co., Ltd. (Shanghai, People's Republic of China), and the sequences were as follows: forward, $5^{\prime}$-AAT GAG TAC CGA AAA TTC-3' and reverse, 5'-CAT CTA GTC CGG ACC GGG AAG-3' for COX-2; forward, 5'-ACC ACA GTC CAT GCC ATC AC-3' and reverse, 5'-TCC ACC ACC CTG TTG CTG TA-3' for GAPDH. COX-2 siRNA was purchased from Shanghai GenePharma Co., Ltd. (Shanghai, People's Republic of China). Lipofectamine 2000 reagent was purchased from Thermo Fisher Scientific (Waltham, MA, USA). The primary antibodies against human COX-2, Bax, Bcl-2, and $\beta$-actin were obtained from Cell Signaling Technology (Beverly, MA, USA). All other chemicals were of reagent grade and obtained from commercial sources.

\section{Cell culture}

All the cell lines were cultured in RPMI 1640 medium supplemented with heat-inactivated $10 \% \mathrm{FBS}, 100 \mathrm{IU}$ penicillin and $100 \mu \mathrm{g} / \mathrm{mL}$ streptomycin in a humidified incubator at $37^{\circ} \mathrm{C}$ and $5 \% \mathrm{CO}_{2}$; transfer of culture was performed once every 3-4 days. When the cells reached logarithmic growth, $0.25 \%$ pancreatin was used to treat the cells for 2 minutes. The digested cells were resuspended using RPMI 1640 medium containing $10 \%$ FBS and then counted, and finally the concentration of cells was adjusted to $1 \times 10^{4}$ cells $/ \mathrm{mL}$.

\section{Cell viability assay}

A 3-(4,5-dimethylthiazol-2-yl)-2,5-diphenyltetrazolium bromide (MTT) assay was used to analyze the viability of cell lines after test agents' treatment. Briefly, all normal and cancer cell lines were seeded into 96-well plates $\left(6 \times 10^{3}\right.$ cells $/$ well $)$ and allowed to attach overnight. After cellular adhesion, the medium was replaced with fresh medium supplemented with various concentrations of test agents and further cultivated for the indicated periods. The control culture received only the culture medium. Following further incubation, MTT was added at a concentration of $5 \mathrm{mg} / \mathrm{mL}$, and the cells were incubated for another 4 hours at $37^{\circ} \mathrm{C}$. After the medium was discarded, dimethyl sulfoxide was added to dissolve MTT formazan crystals. The absorbance reading of each well was determined using a multi-well plate reader at a wavelength of $570 \mathrm{~nm}$. The wells without artesunate and the free cells (culture medium alone) 
were used as background. The cell growth inhibitory rates were defined as the relative absorbance of treated versus untreated cells.

\section{Cell apoptosis assay}

To quantify apoptosis, cells were stained with annexin $\mathrm{V}$ and PI using the annexin V-FITC/PI apoptosis kit according to the manufacturer's instructions. Briefly, gastric cancer cells were cultured in the six-well plates with medium for 24 hours. The cells were then treated for a further 48 hours with test agents. After treatment, the cells were washed twice with cold phosphate-buffered saline (PBS) following treatment and resuspended in $195 \mu \mathrm{L}$ annexin V-FITC binding buffer. Annexin V-FITC ( $5 \mu \mathrm{L})$ was added and mixed gently and the cells were incubated for 15 minutes at room temperature in the dark. The cells were then centrifuged at $1,000 \times g$ for 5 minutes and gently resuspended in $190 \mu \mathrm{L}$ annexin V-FITC binding buffer. Following this, $10 \mu \mathrm{L}$ PI staining solution was added and gently mixed. The cells were kept on ice in the dark and immediately subjected to flow cytometry. FCM Cell Quest software was used to analyze the data.

\section{COX-2 siRNA synthesis and transfection}

Gastric cancer cells $\left(2 \times 10^{5}\right.$ in $2 \mathrm{~mL}$ of RPMI 1640 without antibiotics) were plated in six-well plates. After 24 hours, the human-specific COX-2 siRNA mix with Lipofectamine 2000 was overlaid on the cells according to the manufacturer's protocol. After 48 hours of transfection, cells were harvested for the cell viability assay, the cell apoptosis assay, reverse transcription polymerase chain reaction analysis, and Western blot analysis.

\section{Caspase activity determination}

The caspase- 3 colorimetric assay kit and a caspase- 9 colorimetric assay kit were used to measure the activity of caspase- 3 and caspase- 9 . The assay is based on the cleavage of the chromogenic substrates DEVD-pNA and LEHD-pNA by caspase- 3 and caspase- 9 , respectively. According to the manufacturer's instructions, gastric cells were seeded into 96-well white opaque plates and a corresponding optically clear 96-well plate, and then allowed to adhere overnight. After cellular adhesion, cells were treated with artesunate $(20 \mathrm{mg} / \mathrm{L}, 40 \mathrm{mg} / \mathrm{L}$, and $80 \mathrm{mg} / \mathrm{L})$. At the end of the incubation time, cells were harvested and then lysed in chilled lysis buffer on ice for 10 minutes and centrifuged for 5 minutes at $10,000 \mathrm{rpm}$. Caspase substrate solution containing the specific peptide substrate was then added to the supernatant and incubated for 2 hours at $37^{\circ} \mathrm{C}$. The activities were quantified spectrophotometrically at a wavelength of $405 \mathrm{~nm}$.

\section{Measurement of mitochondrial membrane potential}

Mitochondrial membrane potential was measured by rhodamine 123 staining. Gastric cancer cells were cultured in the six-well plates and allowed to attach overnight. After cellular adhesion, the cells were then treated for a further 48 hours with artesunate, as described previously. Cells were harvested and washed twice with PBS, and then incubated with $20 \mu \mathrm{L}$ rhodamine 123 staining solution at $37^{\circ} \mathrm{C}$ in the dark for 30 minutes, then washed twice with PBS and centrifuged at $500 \times g$ for 10 minutes. Finally, absorbance was determined using a spectrofluorometer at an excitation wavelength of $505 \mathrm{~nm}$ and an emission wavelength of $534 \mathrm{~nm}$.

\section{Western blot assay}

Protein expression levels were analyzed by Western blot. Briefly, gastric cancer cells were seeded in six-well plates at a density of $2.5 \times 10^{5}$ cells and were then incubated overnight at $37^{\circ} \mathrm{C}$ before treatment. After cells were treated with artesunate (20 mg/L, $40 \mathrm{mg} / \mathrm{L}$, and $80 \mathrm{mg} / \mathrm{L}$, respectively) for 48 hours, the cells were washed with PBS and lysed with lysis buffer (20 mM Tris-HCl, pH 7.4, 150 mM NaCl, 0.5\% Nonidet P-40, $1 \mathrm{mM}$ ethylenediaminetetraacetic acid, $50 \mu \mathrm{g} / \mathrm{mL}$ leupeptin, $30 \mu \mathrm{g} / \mathrm{mL}$ aprotinin, and $1 \mathrm{mM}$ PMSF), and incubated at $4^{\circ} \mathrm{C}$ for 1 hour. The extracts were cleared by centrifugation at $13,000 \mathrm{rpm}$ for 20 minutes at $4^{\circ} \mathrm{C}$. The concentration of protein was determined using a bicinchoninic acid protein assay kit according to the manufacturer's instructions. Protein was loaded at a concentration of $40 \mu \mathrm{g}$ per lane, separated on a $12.5 \%$ sodium dodecyl sulfate polyacrylamide gel electrophoresis (SDS-PAGE) gel, and then transferred onto a nitrocellulose membrane using a wet transfer system. Next, the membrane was blocked with $10 \%$ nonfat dry milk in TBST (Tris-buffered saline with Tween 20, pH 8.0) and then incubated with primary antibodies (COX-2, Bax, Bcl-2, and actin) overnight at $4^{\circ} \mathrm{C}$. The appropriate horseradish peroxidase (HRP)-conjugated secondary antibodies were used at 1:3,000 for all antibodies. Positive antibody reactions were detected with the enhanced chemiluminescence system and Hyperfilm X-ray film.

\section{Statistical analysis}

All experimental data are shown as the mean \pm standard deviation. Student's $t$-test was used for comparison of the values between two groups. $P<0.05$ was considered to indicate a statistically significant difference. 


\section{Results}

\section{Effect of artesunate on proliferation}

\section{in gastric cancer cells}

The three human gastric cancer cell lines were treated with $20 \mathrm{mg} / \mathrm{L}$ artesunate, and the viability of cells was assessed by MTT assay from 24 to 72 hours. After treatment, the proliferation of these cell lines was significantly inhibited, especially in HGC-27 cells (Figure 1A). Furthermore, the growth rate of $\mathrm{HGC}-27$ cells was greatly decreased by incubation with 40 and $80 \mathrm{mg} / \mathrm{L}$ artesunate (Figure 1B). The viability of HGC-27 cells treated with artesunate decreased in a dose- and time-dependent manner.

\section{Effect of artesunate on cell apoptosis in gastric cancer cells}

To determine whether the growth-inhibitory effect of artesunate is related to the induction of apoptosis, gastric cancer cells treated with artesunate for 48 hours were analyzed using flow cytometry analysis. As revealed in Figure 2, the proportion of apoptotic cells increased from $12.3 \% \pm 2.1 \%$ to

A

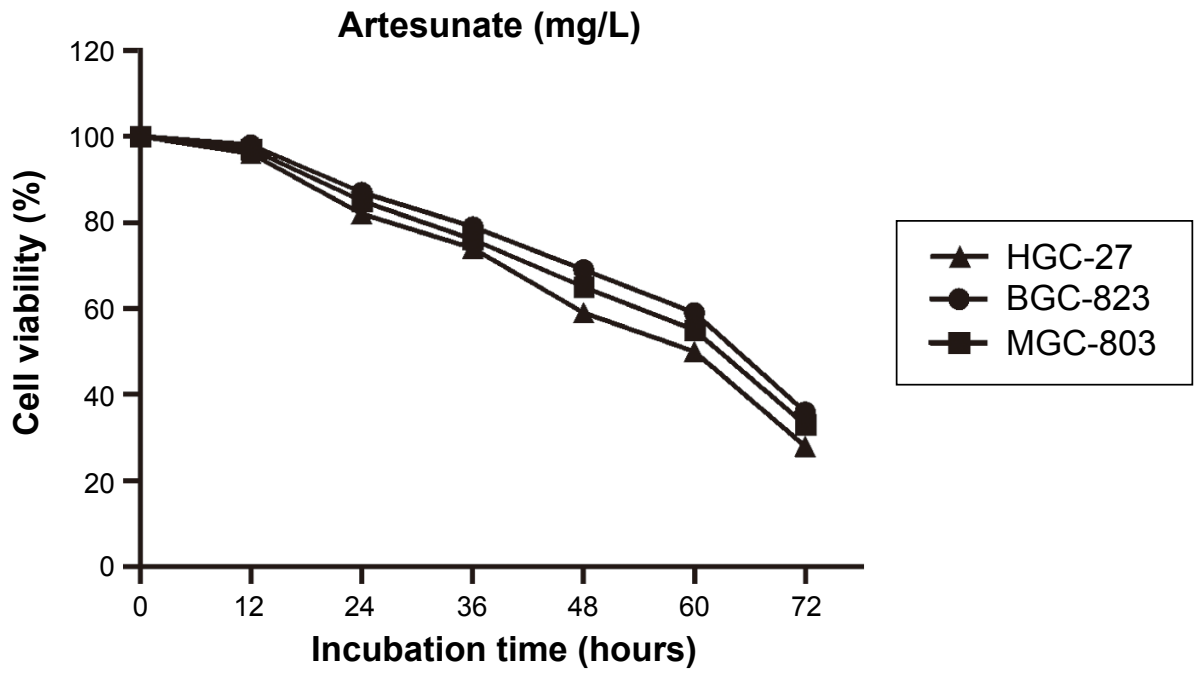

B

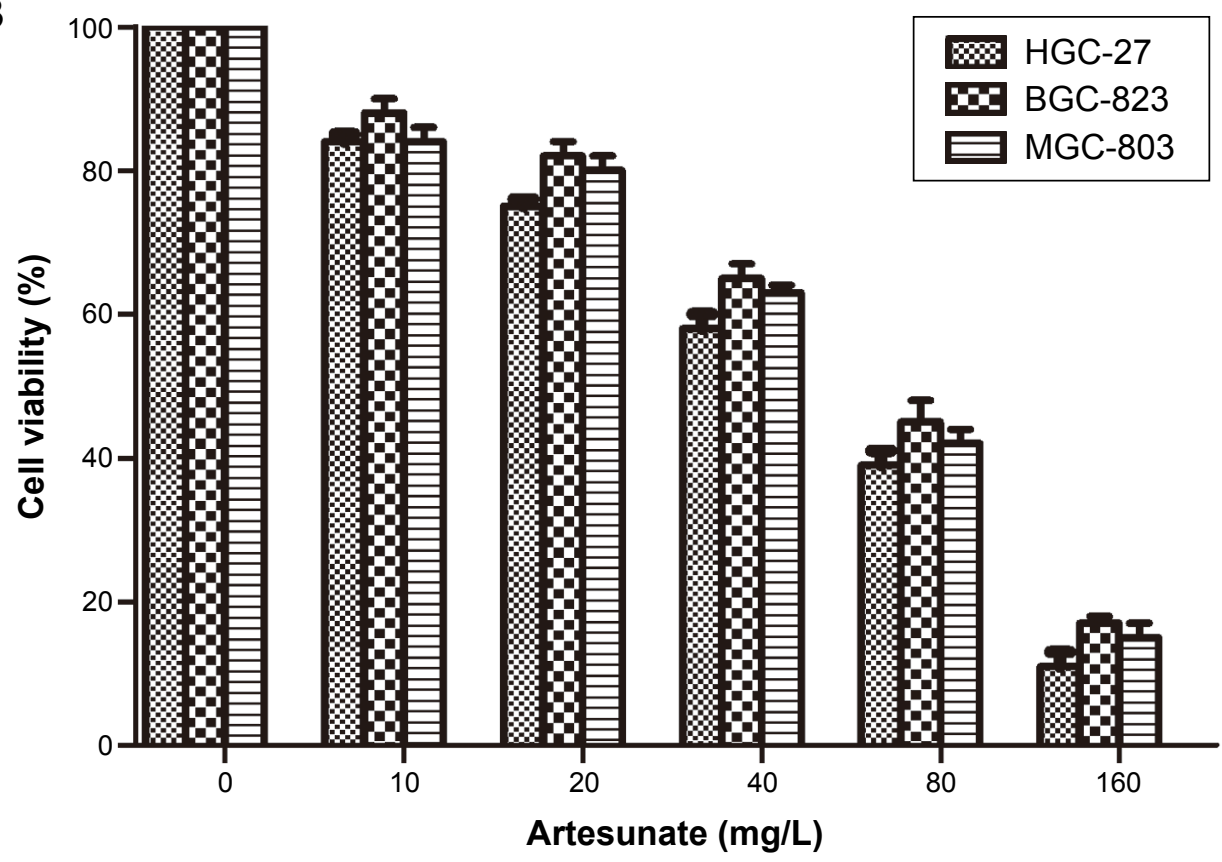

Figure I Artesunate inhibits the proliferation of gastric cancer cells in vitro.

Notes: A 3-(4,5-dimethylthiazol-2-yl)-2,5-diphenyltetrazolium bromide (MTT) assay was used to assess the proliferation of gastric cancer cells. (A) Cell viability of three gastric cancer cell lines, BGC-823, HGC-27, and MGC-803, treated with 40 mg/L artesunate for 0, I2, 24, 36, 48, 60, and 72 hours. (B) Cell viability of gastric cancer cell lines treated with various concentrations of artesunate for 48 hours. 


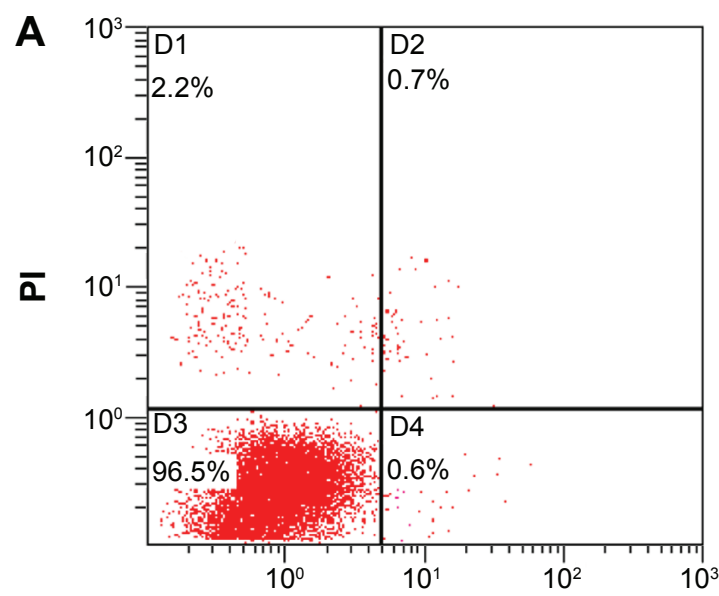

Annexin V-FITC/PI

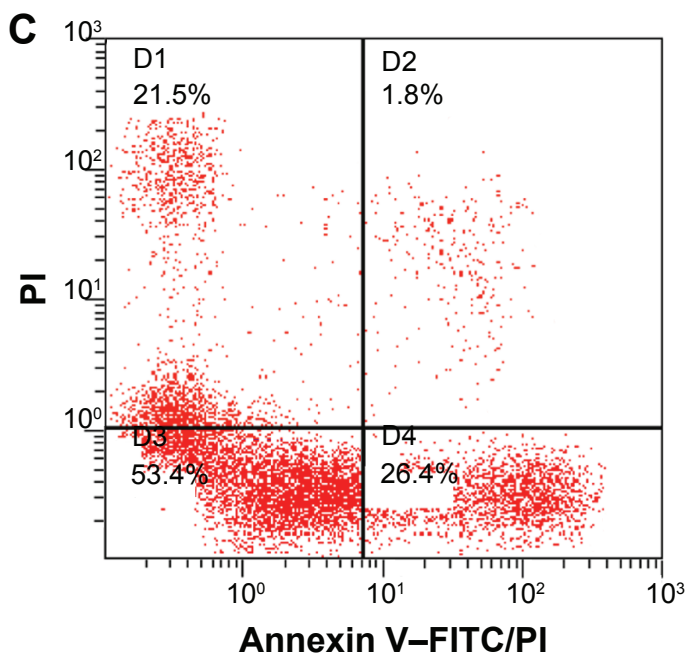

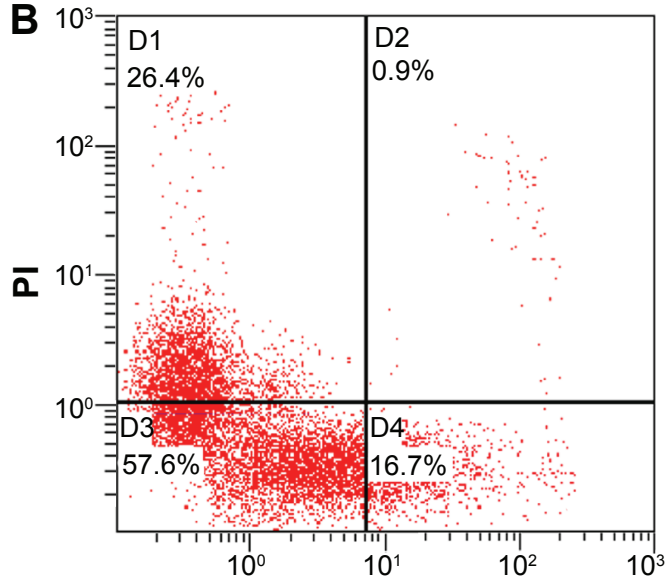

Annexin V-FITC/PI

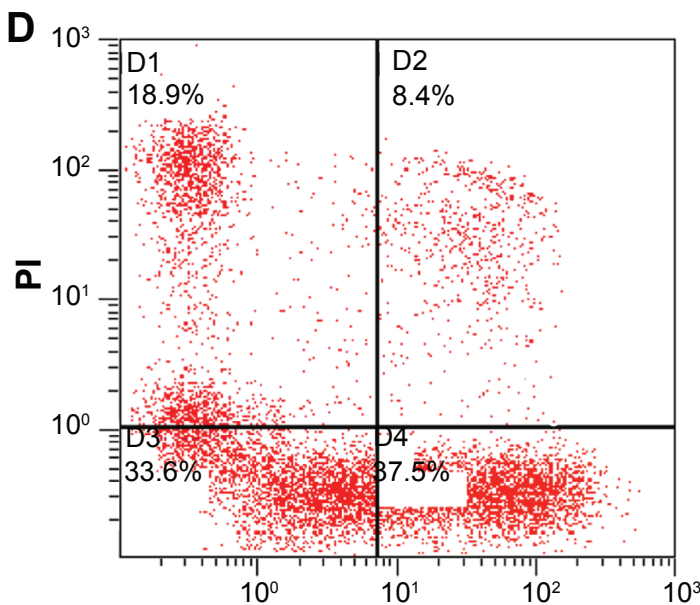

Annexin V-FITC/PI

Figure 2 Artesunate-induced apoptosis in HGC-27 cells.

Notes: HGC-27 cells were treated with the indicated concentrations of artesunate for 48 hours, and the effect of artesunate on cell apoptosis was analyzed by flow cytometry. (A) $0 \mathrm{mg} / \mathrm{L}$. (B) $20 \mathrm{mg} / \mathrm{L}$. (C) $40 \mathrm{mg} / \mathrm{L}$. (D) $80 \mathrm{mg} / \mathrm{L}$. The cells in the D4 quadrant of the histogram represent the number of early apoptotic cells, while those in the $\mathrm{D} 2$ quadrant of the histogram represent the cells in late apoptosis.

Abbreviations: FITC, fluorescein isothiocyanate; PI, propidium iodide.

$35.4 \% \pm 2.8 \%$ in a dose-dependent manner. The percentage of apoptotic cells was significantly higher among those treated with artesunate compared with the control group $(P<0.01)$, indicating that artesunate may inhibit the growth of gastric cancer cells by inducing apoptosis.

\section{Effect of artesunate on expression of COX-2 in gastric cancer cells}

COX-2, overexpressed in various cancers, plays an important role in tumor formation, progression, invasion, and metastasis. To elucidate the interaction between COX-2 and artesunate, HGC-27 cells were exposed to $0,20,40$, and $80 \mathrm{mg} / \mathrm{L}$ artesunate for 48 hours and the expression of COX-2 was assessed using Western blot analysis. As demonstrated in Figure 3, artesunate treatment was associated with reduced expression of COX-2. The expression of COX-2 in cells treated with $20 \mathrm{mg} / \mathrm{L}$ artesunate was observed to be lower than that in the controls. Treatment with $80 \mathrm{mg} / \mathrm{L}$ artesunate led to a further decrease, indicative of a dose-dependent decrease in COX-2 expression.

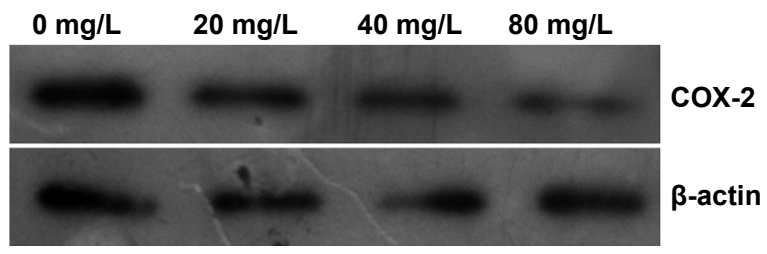

Figure 3 Artesunate suppressed the expression of COX-2.

Notes: The levels of COX-2 were determined in cell lysates using Western blot analysis. Treatment of HGC-27 cells with artesunate resulted in downregulation of COX-2 expression. 


\section{Effect of celecoxib, a selective COX-2 inhibitor, on cell proliferation and apoptosis in gastric cancer cells}

To explore whether the antitumor effect of artesunate on gastric cancer cells is mediated through its inhibitory effect on COX-2 expression, the viability of HGC-27 cells was assessed by MTT assay after treatment with various concentrations of celecoxib $(0,20,40$, and $80 \mu \mathrm{mol} / \mathrm{L})$, a well-known inhibitor of COX-2, from 24 to 72 hours. Treatment of the cells with celecoxib resulted in a dose- and time-dependent reduction in the cell viability of HGC-27 cells as compared with non-celecoxib-treated controls $(P<0.05)$ (Figure 4$)$. Celecoxib also could induce cell apoptosis (Figure 5). Treatment of the HGC-27 cells with celecoxib for 48 hours induced a marked, dose-dependent induction of both the early and late stages of apoptosis. Celecoxib treatment increased the number of apoptotic cells from $8.83 \%$ in the untreated cell group to $25.86 \%$ in the group treated with $80 \mu \mathrm{mol} / \mathrm{L}$ celecoxib. These data suggest that the inhibition of COX-2 expression is linked to the inhibition of cell proliferation and induction of cell apoptosis.

\section{Effect of siRNA knockdown of COX-2 on cell proliferation and apoptosis}

We further verified the role of COX-2 in cell proliferation and apoptosis through siRNA knockdown of COX-2 in the gastric cells and examined whether it would lead to the inhibition of cell proliferation and the induction of cell apoptosis. After 48 hours of transfection, the expression of COX-2 was analyzed by reverse transcription polymerase chain reaction and Western blot analysis. The mRNA expression and protein

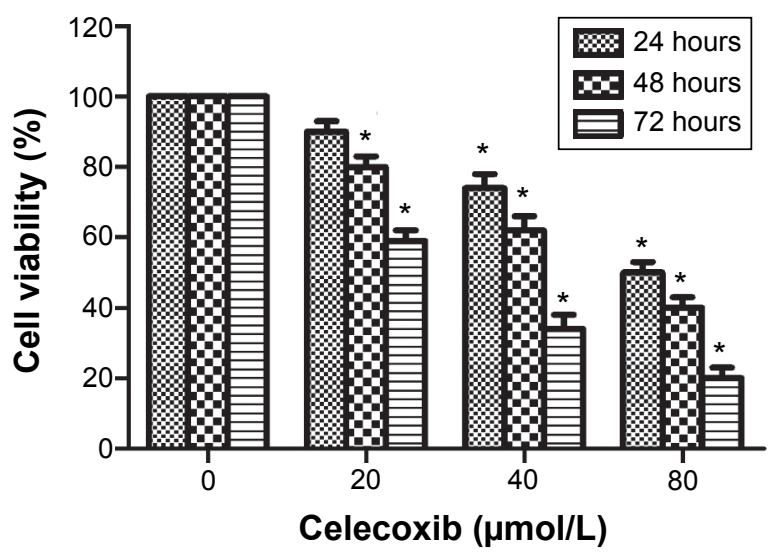

Figure 4 Celecoxib, a selective COX-2 inhibitor, inhibits cell proliferation in HGC-27 cells.

Notes: The viability of HGC-27 cells treated with the indicated concentrations of celecoxib for $0,24,48$, and 72 hours is shown. Treatment with celecoxib caused a dose- and time-dependent inhibition of cell proliferation. $* P<0.05$ versus $0 \mu \mathrm{mol} / \mathrm{L}$. expression levels of COX-2 were significantly decreased in the COX-2 siRNA group compared with the control siRNA group (Figure 6A). The transfection of HGC-27 cells with COX-2 siRNA resulted in a significant reduction in the cell proliferation of HGC-27 cells after 48 hours as compared to that of the control siRNA-transfected HGC-27 cells (Figure 6B). We also analyzed the effect of COX-2 siRNA on cell apoptosis in HGC-27 cells using annexin V-FITC/ PI staining. The transfection of HGC-27 cells with COX-2 siRNA increased the number of apoptotic cells from 5.55\% in the control siRNA-transfected cell group to $19.35 \%$ in the COX-2 siRNA group (Figure 6C).

\section{Effect of artesunate on caspase activity and the protein expression of Bax and $\mathrm{Bcl}-2$ in gastric cancer cells}

To examine the mechanism of apoptosis induced by artesunate in gastric cancer cells, we analyzed mitochondrial features of the intrinsic apoptotic pathway. The proapoptotic Bcl-2 family members, such as Bax and Bcl-2, are essential for the initiation of mitochondrial dysfunction during apoptosis. The protein expression levels of Bax and Bcl-2 were assessed by Western blot analysis. Results showed that, in HGC-27 cells, treatment with increased doses of artesunate led to increased expression of Bax and decreased expression of antiapoptotic Bcl-2 (Figure 7A). In addition, the activity of caspase-3 and caspase-9 was upregulated (Figure 7B).

\section{Effect of artesunate on mitochondrial membrane potential in gastric cancer cells}

A large number of studies demonstrate that the disruption of mitochondrial integrity is a critical step occurring in cells undergoing apoptosis. A decreasing mitochondrial membrane potential is related to mitochondrial dysfunction, and loss of mitochondrial membrane potential plays a vital role in the mitochondrial-mediated apoptosis. As shown in Figure 8, after treatment with various concentrations of artesunate for 48 hours, the fluorescence intensity was significantly decreased in the HGC-27 cells, suggesting that artesunate treatment of gastric cancer cells induces apoptosis through the mitochondrial apoptosis pathway.

\section{Discussion}

Gastric cancer is the second most common cause of cancerrelated mortalities worldwide and the fourth most common cancer, with approximately 900,000 new cases and 700,000 deaths per year. ${ }^{19}$ In East Asian countries especially, like the People's Republic of China and Japan, more than 1 million 
A

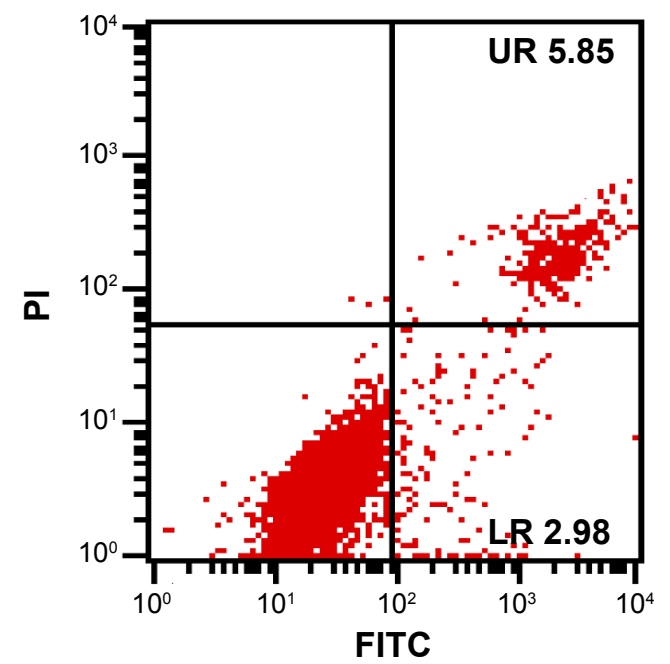

C

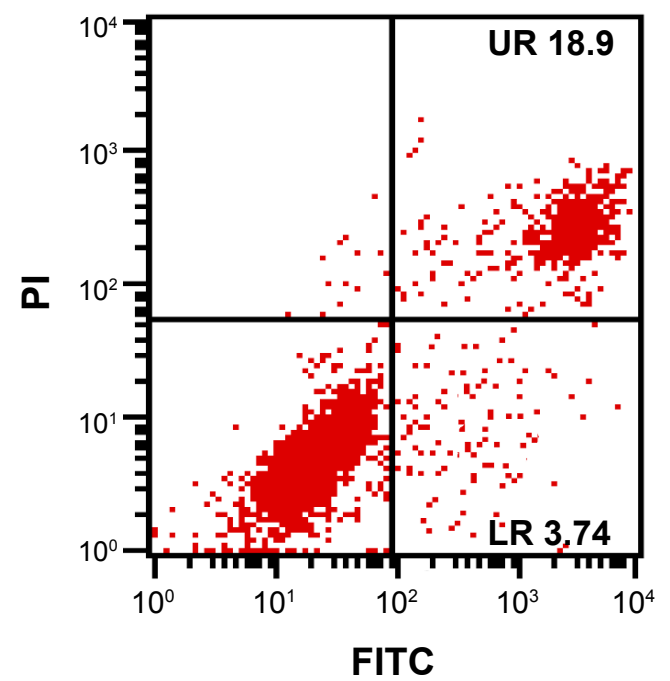

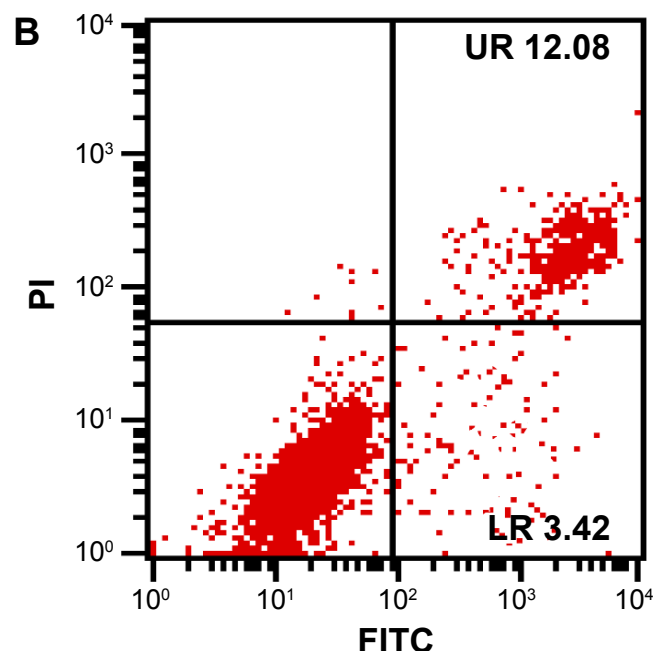

FITC

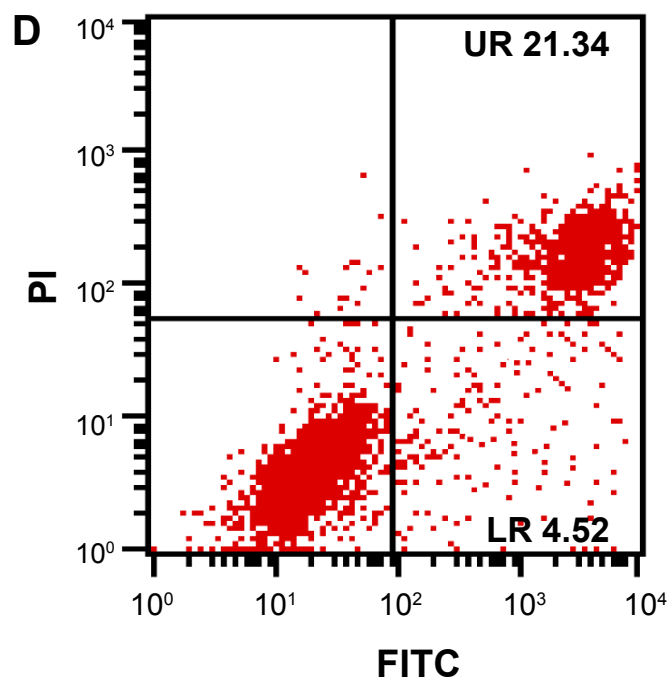

Figure 5 Celecoxib induces apoptosis in HGC-27 cells.

Notes: HGC-27 cells were treated with the indicated concentrations of artesunate for 48 hours, and annexin V-FITC/PI staining was performed. (A) 0, (B) 20, (C) 40, and (D) $80 \mu \mathrm{mol} / \mathrm{L}$ celecoxib. The cells in the LR quadrant of each histogram represent the number of early apoptotic cells, while those in the UR quadrant of each histogram represent the cells in late apoptosis.

Abbreviations: FITC, fluorescein isothiocyanate; LR, lower right; PI, propidium iodide; UR, upper right.

new cases are diagnosed with gastric cancer each year. ${ }^{20}$ Attempts to improve the outcomes of this disease have incorporated the use of adjuvant chemotherapy. However, the clinical applications of standard anticancer drugs such as adriamycin, 5-fluorouracil, and cisplatin are limited because of the severe side effects. As a result, increasing attention has been focused on the application of natural products in the treatment of gastric cancer.

Artesunate has been traditionally used to treat malaria, especially against cerebral malaria. Previous studies have revealed that artesunate possesses a number of biological activities ${ }^{21,22}$ and exhibits antiproliferative effects and apoptosis-inducing activities in various tumor cell lines in vitro and in vivo. ${ }^{23-25}$ Although numerous studies have demonstrated that artesunate may have potential as an anticancer therapy, there is little available information about the antitumor effects of artesunate on human gastric cancer cells. In the present study, our results show that artesunate effectively inhibited HGC-27, BGC823, and MGC803 cell proliferation, especially in HGC-27 cells, and all the effects were in a time and dose dependent manner. It also revealed that artesunate resulted in apoptosis of treated cells in a dose-dependent manner. These results indicate that artesunate may inhibit the proliferation of cultured HGC-27 cells by activating the apoptotic signaling pathway.

Apoptosis, a tightly regulated signaling process that involves the coordination of both antiapoptotic and proapoptotic proteins, is vital for anti-carcinogenesis. ${ }^{26}$ The proapoptotic Bcl-2 family members, such as Bax and Bcl-2, are essential for the initiation of mitochondrial dysfunction during apoptosis. 

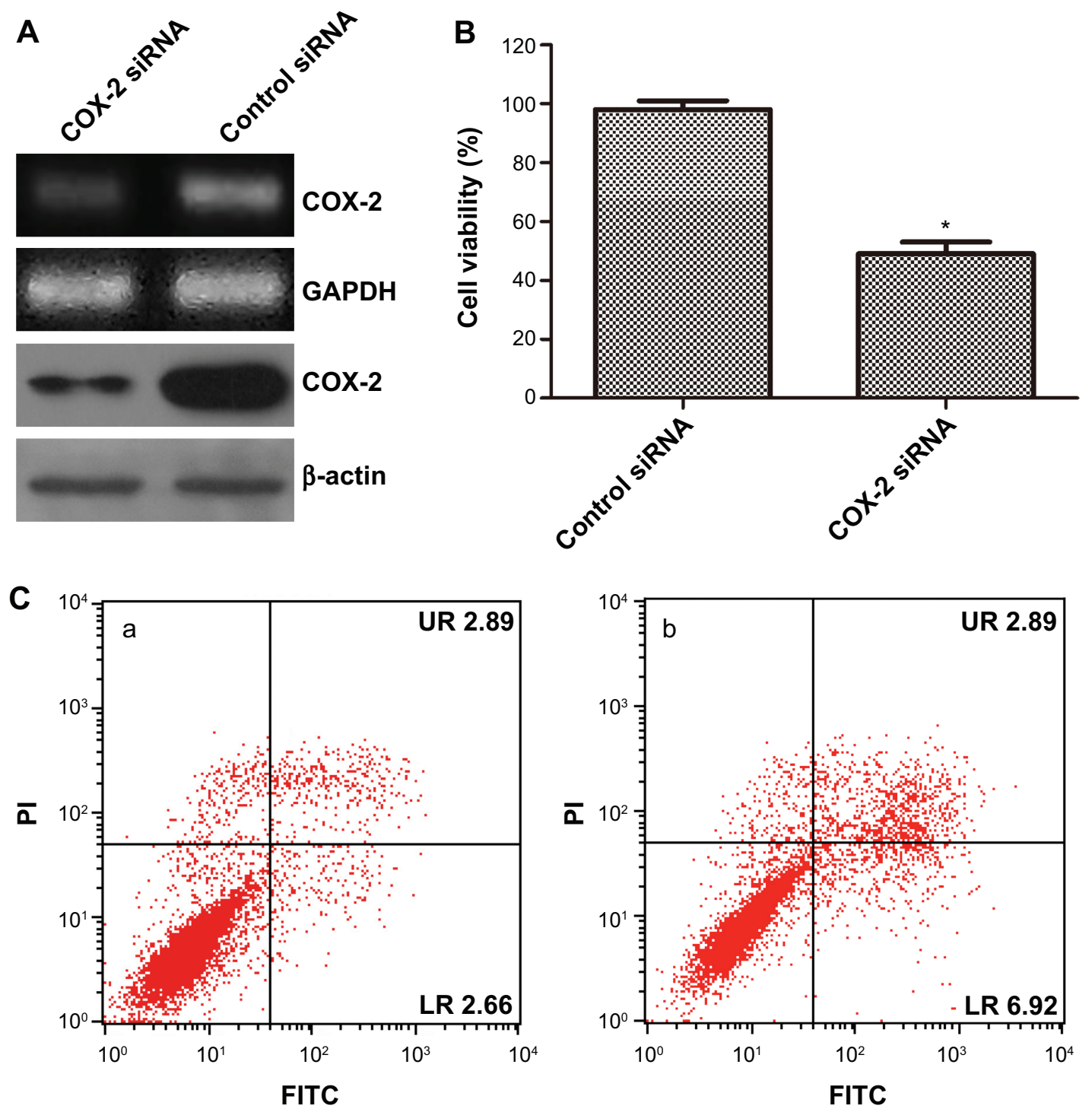

Figure 6 siRNA knockdown of COX-2 leads to inhibition of cell proliferation and induction of cell apoptosis.

Notes: (A) COX-2 expression was analyzed by RT-PCR analysis and Western blotting in HGC-27 cells and COX-2 siRNA-transfected HGC-27 cells. (B) Forty-eight hours after transfection, the proliferation of HGC-27 cells was significantly inhibited. $* P<0.05$ versus control siRNA-transfected HGC-27 cells. (C) The apoptosis-inducing effect was analyzed by flow cytometry after 48 hours of transfection in control siRNA-transfected cell group (a) and COX-2 siRNA-transfected cell group (b).

Abbreviations: FITC, fluorescein isothiocyanate; LR, lower right; PI, propidium iodide; RT-PCR, reverse transcription polymerase chain reaction; UR, upper right.

The results show that the expression of proapoptotic factor Bax was markedly upregulated in the artesunate treatment group. However, antiapoptotic factor Bcl-2 was reduced significantly. Also, artesunate treatment led to an increase in the activity of caspase-3 and caspase-9. Artesunate could also induce loss of mitochondrial membrane potential in HGC-27 cells. These results indicate that artesunate could induce apoptosis in gastric cancer cells by activating the mitochondrial apoptosis pathway.

$\mathrm{COX}-2$, the inducible enzyme that regulates prostaglandin E2 (PGE2) synthesis, is frequently overexpressed in various cancers. ${ }^{27,28} \mathrm{COX}-2$ and PGE2 play a central role in orchestrating the multiple events of cancer invasion, metastasis, and tumor development. ${ }^{29-31}$ Moreover, a metaanalysis demonstrated that high COX-2 expression may be an independent risk factor for poor overall survival of patients with gastric cancer. ${ }^{32}$ Additionally, early-stage gastric cancer patients with high expression of COX-2 protein were at a higher risk for cancer-related death than those with a low level of COX-2 expression. ${ }^{33}$ Therefore, COX-2 is considered a promising target for cancer therapy. ${ }^{3}$ The search for novel and low-toxicity inhibitors of COX-2 may provide a better option for the treatment of gastric cancer and that may provide a better strategy for its prevention or treatment. In the present study, artesunate was shown to suppress the expression of COX-2 in HGC-27 cells in 
A

$0 \mathrm{mg} / \mathrm{L} \quad 20 \mathrm{mg} / \mathrm{L} \quad 40 \mathrm{mg} / \mathrm{L} \quad 80 \mathrm{mg} / \mathrm{L}$
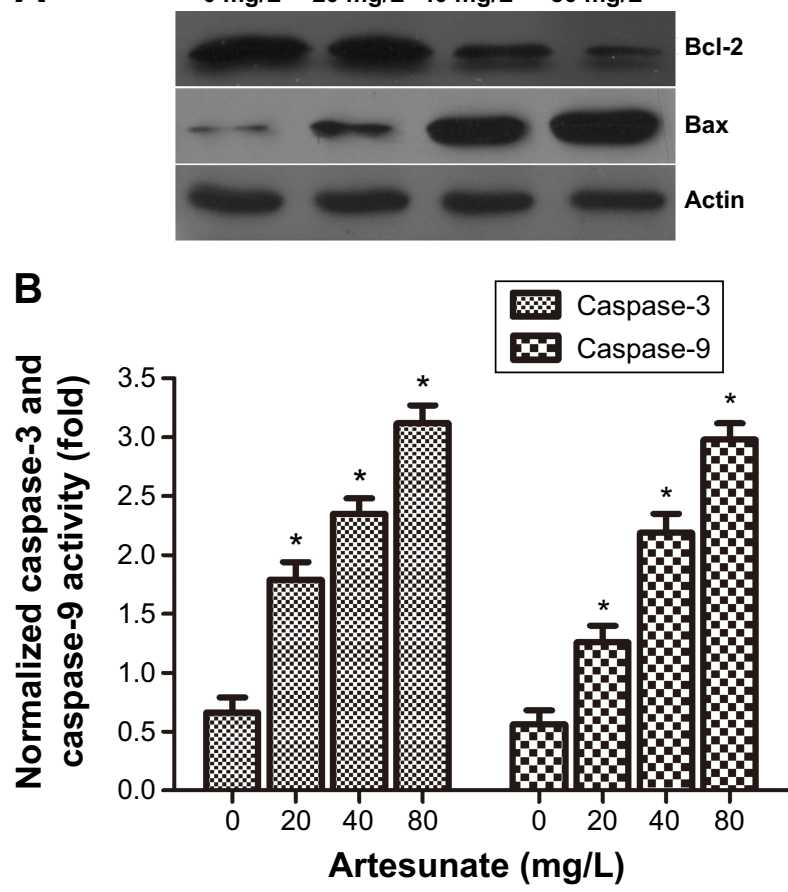

Figure 7 Effects of artesunate on the expression of proapoptotic and antiapoptotic elements in HGC-27 cells.

Notes: HGC-27 cells were treated with $0,20,40$, and $80 \mathrm{mg} / \mathrm{L}$ artesunate for 48 hours. (A) Artesunate promoted the expressions of Bax, but decreased $\mathrm{Bcl}-2$ expression. (B) Analysis of relative caspase- 3 and caspase- 9 activity in cells. Artesunate increased the activity of caspase- 3 and caspase- 9 . $* P<0.05$ versus $0 \mathrm{mg} / \mathrm{L}$.

a dose-dependent manner. These results indicate that the effects of artesunate on cell growth inhibition and apoptosis were associated with the inhibition of COX-2 expression. This was supported by evidence that treatment of HGC-27 cells with celecoxib, a selected COX-2 inhibitor, resulted in a reduction in the cell viability and an increase in cell apoptosis. Similar effects were noted when the HGC-27 cells

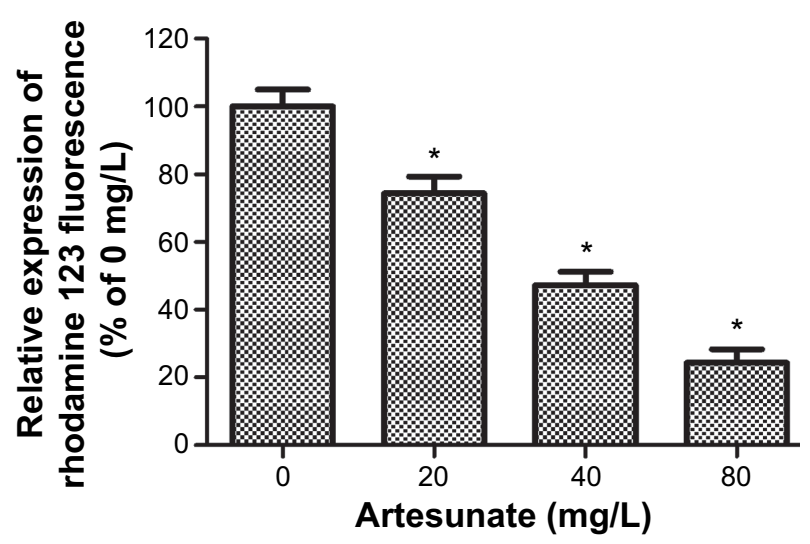

Figure 8 Artesunate reduced the rhodamine 123 fluorescence intensity in HGC-27 cells treated with $0,20,40$, and $80 \mathrm{mg} / \mathrm{L}$ artesunate for 48 hours.

Notes: The results suggest artesunate could reduce the mitochondrial membrane potential and artesunate treatment of gastric cancer cells induces apoptosis through the mitochondrial apoptosis pathway. ${ }^{*} P<0.05$ versus $0 \mathrm{mg} / \mathrm{L}$. were transfected with COX-2 siRNA. By stimulating the growth of blood vessels, inhibiting local immune function, and regulating a variety of signal transduction pathways, PGE2 contributes to promoting the proliferation of cancer cells and the growth of tumors, reducing apoptosis, and increasing tumor invasiveness. ${ }^{34-36}$ The protein encoded by COX-2 genes is a type of oncogenic protein, which could catalyze the conversion of arachidonic acid to PGE2. Some studies confirmed that overexpression of COX-2 promoted cell proliferation by weakening the antiproliferative effect of transforming growth factor- $\beta$ (TGF- $\beta$ ) ${ }^{37}$ Previous study demonstrated that COX-2 was a regulatory factor in the Bcl-2 upstream sequences through the phosphatidylinositol 3-kinase (PI3K) signal pathway, and eventually inhibited the apoptosis of cancer cells. ${ }^{38}$ It has also been confirmed that COX-2 could inhibit the apoptosis of cancer cells by inducing the mutation of P53 and weakening the apoptotic signal mediated by Fas protein. ${ }^{39}$ These results indicate that artesunate induces apoptosis by suppressing the expression of COX-2, which led to downregulation of Bcl-2 and upregulation of Bax.

In summary, the results from this study suggest that artesunate exhibits a marked antitumor effect. One of the antitumor mechanisms of artesunate may be that its inhibition of COX-2 led to reduced proliferation and induction of apoptosis, connected with mitochondrial dysfunction. These results provide a rationale to continue research with artesunate, and further mechanism-based in vivo studies are required that can establish the importance of artesunate and its development as a pharmacologically safe, nontoxic agent for gastric cancer treatment by use either alone or in combination with conventional therapeutics.

\section{Disclosure}

The authors report no conflicts of interest in this work.

\section{References}

1. Siegel R, Naishadham D, Jemal A. Cancer statistics, 2013. CA Cancer J Clin. 2013;63(1):11-30.

2. Bornschein J, Rokkas T, Selgrad M, Malfertheiner P. Gastric cancer: clinical aspects, epidemiology and molecular background. Helicobacter. 2011;16 Suppl 1:45-52.

3. Méric JB, Rottey S, Olaussen K, et al. Cyclooxygenase-2 as a target for anticancer drug development. Crit Rev Oncol Hematol. 2006;59(1): 51-64.

4. Riedl K, Krysan K, Põld M, et al. Multifaceted roles of cyclooxygenase-2 in lung cancer. Drug Resist Updat. 2004;7(3):169-184.

5. Tucker ON, Dannenberg AJ, Yang EK, et al. Cyclooxygenase-2 expression is up-regulated in human pancreatic cancer. Cancer Res. 1999;59(5): 987-990.

6. Cheng J, Fan XM. Role of cyclooxygenase-2 in gastric cancer development and progression. World J Gastroenterol. 2013;19(42):7361-7368. 
7. Bocca C, Ievolella M, Autelli R, et al. Expression of Cox-2 in human breast cancer cells as a critical determinant of epithelial-to-mesenchymal transition and invasiveness. Expert Opin Ther Targets. 2014;18(2): 121-135.

8. Elder DJ, Halton DE, Crew TE, Paraskeva C. Apoptosis induction and cyclooxygenase-2 regulation in human colorectal adenoma and carcinoma cell lines by the cyclooxygenase- 2 selective non-steroidal anti-inflammatory drug NS-398. Int J Cancer. 2000;86(4):553-560.

9. Solomon SD, Wittes J, Finn PV, et al. Cardiovascular risk of celecoxib in 6 randomized placebo-controlled trials: the cross trial safety analysis. Circulation. 2008;117(16):2104-2113.

10. Cui X, Wang Y, Kokudo N, Fang D, Tang W. Traditional Chinese medicine and related active compounds against hepatitis B virus infection. Biosci Trends. 2010;4(2):39-47.

11. Jiang W, Li B, Zheng $X$, et al. Artesunate in combination with oxacillin protect sepsis model mice challenged with lethal live methicillin-resistant Staphylococcus aureus (MRSA) via its inhibition on proinflammatory cytokines release and enhancement on antibacterial activity of oxacillin. Int Immunopharmacol. 2011;11(8):1065-1073.

12. Yang Z, Ding J, Yang C, et al. Immunomodulatory and anti-inflammatory properties of artesunate in experimental colitis. Curr Med Chem. 2012; 19(26):4541-4551.

13. Cheng C, Ng DS, Chan TK, et al. Anti-allergic action of anti-malarial drug artesunate in experimental mast cell-mediated anaphylactic models. Allergy. 2013;68(2):195-203.

14. Shi JQ, Zhang CC, Sun XL, et al. Antimalarial drug artemisinin extenuates amyloidogenesis and neuroinflammation in APPswe/PS1dE9 transgenic mice via inhibition of nuclear factor- $\mathrm{KB}$ and NLRP3 inflammasome activation. CNS Neurosci Ther. 2013;19(4):262-268.

15. Li T, Chen H, Yang Z, Liu XG, Zhang LM, Wang H. Evaluation of the immunosuppressive activity of artesunate in vitro and in vivo. Int Immunopharmacol. 2013;16(2):306-312.

16. Bachmeier B, Fichtner I, Killian PH, Kronski E, Pfeffer U, Efferth T. Development of resistance towards artesunate in MDA-MB-231 human breast cancer cells. PLoS One. 2011;6(5):e20550.

17. Jin M, Shen X, Zhao C, et al. In vivo study of effects of artesunate nanoliposomes on human hepatocellular carcinoma xenografts in nude mice. Drug Deliv. 2013;20(3-4):127-133.

18. Du JH, Zhang HD, Ma ZJ, Ji KM. Artesunate induces oncosis-like cell death in vitro and has antitumor activity against pancreatic cancer xenografts in vivo. Cancer Chemother Pharmacol. 2010;65(5):895-902.

19. Bang YJ, Kim YW, Yang HK, et al; CLASSIC trial investigators. Adjuvant capecitabine and oxaliplatin for gastric cancer after D2 gastrectomy (CLASSIC): a phase 3 open-label, randomised controlled trial. Lancet. 2012;379(9813):315-321.

20. Jian P, Yanfang T, Zhuan Z, Jian W, Xueming Z, Jian N. MMP28 (epilysin) as a novel promoter of invasion and metastasis in gastric cancer. BMC Cancer. 2011;11:200.

21. Schreiber A, Härter G, Schubert A, Bunjes D, Mertens T, Michel D. Antiviral treatment of cytomegalovirus infection and resistant strains. Exp Opin Pharmacother. 2009;10(2):191-209.

22. Ho WE, Peh HY, Chan TK, Wong WS. Artemisinins: pharmacological actions beyond anti-malarial. Pharmacol Ther. 2014;142(1): $126-139$.
23. Zhou C, Pan W, Wang XP, Chen TS. Artesunate induces apoptosis via a Bak-mediated caspase-independent intrinsic pathway in human lung adenocarcinoma cells. J Cell Physiol. 2012;227(12):3778-3786.

24. Berdelle N, Nikolova T, Quiros S, Efferth T, Kaina B. Artesunate induces oxidative DNA damage, sustained DNA double-strand breaks, and the ATM/ATR damage response in cancer cells. Mol Cancer Ther. 2011;10(12):2224-2233.

25. Sertel S, Eichhorn T, Simon CH, Plinkert PK, Johnson SW, Efferth T. Pharmacogenomic identification of c-Myc/Max-regulated genes associated with cytotoxicity of artesunate towards human colon, ovarian and lung cancer cell lines. Molecules. 2010;15(4):2886-2910.

26. Tang D, Lotze MT, Kang R, Zeh HJ. Apoptosis promotes early tumorigenesis. Oncogene. 2011;30(16):1851-1854.

27. Masferrer JL, Leahy KM, Koki AT, et al. Antiangiogenic and antitumor activities of cyclooxygenase-2 inhibitors. Cancer Res. 2000;60(5): 1306-1311.

28. Sheng H, Shao J, Kirkland SC, et al. Inhibition of human colon cancer cell growth by selective inhibition of cyclooxygenase-2. J Clin Invest. 1997;99(9):2254-2259.

29. Singh T, Vaid M, Katiyar N, Sharma S, Katiyar SK. Berberine, an isoquinoline alkaloid, inhibits melanoma cancer cell migration by reducing the expressions of cyclooxygenase-2, prostaglandin $\mathrm{E}_{2}$ and prostaglandin $\mathrm{E}_{2}$ receptors. Carcinogenesis. 2011;32(1):86-92.

30. Reich R, Martin GR. Identification of arachidonic acid pathways required for the invasive and metastatic activity of malignant tumor cells. Prostaglandins. 1996;51(1):1-17.

31. Dubois RN, Abramson SB, Crofford L, et al. Cyclooxygenase in biology and disease. FASEB J. 1998;12(12):1063-1073.

32. Song J, Su H, Zhou YY, Guo LL. Cyclooxygenase-2 expression is associated with poor overall survival of patients with gastric cancer: a meta-analysis. Dig Dis Sci. 2014;59(2):436-445.

33. Mrena J, Wiksten JP, Thiel A, et al. Cyclooxygenase- 2 is an independent prognostic factor in gastric cancer and its expression is regulated by the messenger RNA stability factor HuR. Clin Cancer Res. 2005;11(20): 7362-7368.

34. Akhtar M, Cheng Y, Magno RM, et al. Promoter methylation regulates Helicobacter pylori-stimulated cyclooxygenase-2 expression in gastric epithelial cells. Cancer Res. 2001;61(6):2399-2403.

35. Sheng H, Shao J, Washington MK, DuBois RN. Prostaglandin E2 increases growth and motility of colorectal carcinoma cells. J Biol Chem. 2001;276(21):18075-18081.

36. Moalic S, Liagre B, Le Bail JC, Beneytout JL. Dose-dependent modulation of apoptosis and cyclooxygenase-2 expression in human 1547 osteosarcoma cells by NS-398, a selective cyclooxygenase- 2 inhibitor. Int J Oncol. 2001;18(3):533-540.

37. Enders GA. Cyclooxygenase- 2 overexpression abrogates the antiproliferative effects of TGF-beta. Br J Cancer. 2007;97(10):1388-1392.

38. Chen XL, Su BS, Sun RQ, Zhang J, Wang YL. Relationship between expression and distribution of cyclooxygenase-2 and bcl-2 in human gastric adenocarcinoma. World J Gastroenterol. 2005;11(8): 1228-1231.

39. Han JA, Kim JI, Ongusaha PP, et al. P53-mediated induction of Cox-2 counteracts p53- or genotoxic stress-induced apoptosis. EMBO J. 2002;21(21):5635-5644.
OncoTargets and Therapy

\section{Publish your work in this journal}

OncoTargets and Therapy is an international, peer-reviewed, open access journal focusing on the pathological basis of all cancers, potential targets for therapy and treatment protocols employed to improve the management of cancer patients. The journal also focuses on the impact of management programs and new therapeutic agents and protocols on

\section{Dovepress}

patient perspectives such as quality of life, adherence and satisfaction. The manuscript management system is completely online and includes a very quick and fair peer-review system, which is all easy to use. Visit http://www.dovepress.com/testimonials.php to read real quotes from published authors. 